\title{
Utilization of genetic testing among children with developmental disabilities in the United States
}

\section{Bridget Kiely \\ Sujit Vettam \\ Andrew Adesman}

Division of Developmental and Behavioral Pediatrics, Department of Pediatrics, Steven and Alexandra Cohen Children's Medical Center of New York, New Hyde Park, NY, USA
Correspondence: Andrew Adesman Division of Developmental and Behavioral Pediatrics, Department of Pediatrics, Steven and Alexandra Cohen Children's Medical Center of New York, 1983 Marcus Avenue, Suite 130, Lake Success, New York, NY 1 1042, USA

Tel +l 5168026100

Email AAdesman@Northwell.edu
This article was published in the following Dove Press journal:

The Application of Clinical Genetics

II July 2016

Number of times this article has been viewed

Purpose: Several professional societies recommend that genetic testing be routinely included in the etiologic workup of children with developmental disabilities. The aim of this study was to determine the rate at which genetic testing is performed in this population, based on data from a nationally representative survey.

Methods: Data were analyzed from the Survey of Pathways to Diagnosis and Services, a telephone-based survey of parents and guardians of US school-age children with current or past developmental conditions. This study included 3,371 respondents who indicated that their child had an autism spectrum disorder (ASD), intellectual disability (ID), and/or developmental delay (DD) at the time of survey administration. History of genetic testing was assessed based on report by the parent/s. Children were divided into the following five mutually exclusive condition groups: ASD with ID; ASD with DD, without ID; ASD only, without ID or DD; ID without ASD; and DD only, without ID or ASD. Logistic regression was used to assess the demographic correlates of genetic testing, to compare the rates of genetic testing across groups, and to examine associations between genetic testing and use of other health-care services.

Results: Overall, $32 \%$ of this sample had a history of genetic testing, including $34 \%$ of all children with ASD and $43 \%$ of those with ID. After adjusting for demographics, children with ASD + ID were more than seven times as likely as those with ASD only, and more than twice as likely as those who had ID without ASD, to have undergone genetic testing. Prior specialist care (developmental pediatrician or neurologist) and access to all needed providers within the previous year were associated with higher odds of genetic testing.

Conclusion: The majority of children in this nationally representative sample did not undergo recommended genetic testing. Research is needed to identify barriers to the use of genetic testing in this population.

Keywords: autism spectrum disorders, intellectual disability, developmental delay, genetic screening

\section{Introduction}

The prevalence of developmental disabilities in the US has risen in recent years. ${ }^{1}$ An estimated $1 \%-2 \%$ of US children are affected by autism spectrum disorders (ASDs), which are characterized by deficits in communication and social interaction, along with abnormal, stereotyped behaviors, and/or restricted interests that become apparent early in life. ${ }^{2}$ ASDs frequently co-occur with intellectual disability (ID), a condition defined by childhood-onset impairments in general mental abilities associated with an intelligence quotient of $<70$ and adaptive limitations across multiple domains. ${ }^{3}$ Children younger than 5-6 years of age, in whom intelligence quotient and adaptive

submit your manuscript | www.dovepress.con 
functioning are hard to assess, may instead be diagnosed with global developmental delay (GDD) if they demonstrate significant delays in two or more areas. ${ }^{4}$ The prevalence of GDD/ID in US population has been estimated to range from $1 \%$ to $3 \%{ }^{4-6}$

Decades of research have yielded important insights in the etiology of these disorders. Although several environmental exposures have been identified as risk factors for these conditions, findings from twin concordance studies have shown that both ASD and GDD/ID are highly heritable. ${ }^{7}$ However, the genetic basis of these conditions is heterogeneous and includes both copy number and single-nucleotide changes. ${ }^{6}$ Advances in genomic technologies such as chromosomal microarray (CMA) and massively parallel sequencing have facilitated the identification of more than 100 putatively causal genetic changes in individuals with ASD and/or GDD/ ID. ${ }^{8}$ A number of these variants have also been implicated in other neurodevelopmental disorders, including attentiondeficit/hyperactivity disorder, epilepsy, and schizophrenia. ${ }^{6}$

As research about the genetic basis of these conditions has advanced, a number of professional societies have recognized genetic testing as an essential component of the etiologic workup for children with ASD and/or GDD/ID. Guidelines published in the early 2000s by the American Academy of Pediatrics (AAP), the American Academy of Neurology, and the American College of Medical Genetics (ACMG) recommended the use of karyotyping and Fragile $X$ testing as first-tier diagnostic tests for children with unexplained ID/GDD with or withoutASD. ${ }^{5,9-12}$ However, more recent guidelines have revised these recommendations and have called for CMA to replace karyotyping as the first-tier test for detecting chromosomal imbalances. ${ }^{13-15}$ Most of the current guidelines recommend that all children with unexplained ID, GDD, and/or ASD be offered genetic testing, including CMA and Fragile X testing (routinely in males only per ACMG guidelines and in both sexes per AAP guidelines). ${ }^{13-16}$ Other studies - including karyotyping, metabolic or mitochondrial testing, and targeted testing for single-gene disorders other than Fragile $\mathrm{X}$ - may be indicated if the physical examination or family history is suggestive of a specific diagnosis or if initial testing fails to establish a genetic cause. ${ }^{14,15}$ Although the etiologic yield depends on several factors, this approach may result in the identification of an underlying genetic abnormality in up to $40 \%$ of patients. ${ }^{14}$

The potential benefits of routinely performing genetic testing in this population of children are numerous. Determining the etiology of a child's developmental disabilities may facilitate accurate recurrence risk counseling, alleviate parental guilt, and enable the child to avoid unnecessary additional diagnostic testing. ${ }^{17}$ Identification of the genetic basis of a child's condition may also allow clinicians to provide affected children with focused medical surveillance for associated conditions and to direct families to syndrome-specific resources for clinical care, research, and support. ${ }^{15,17-19}$ Nonetheless, the value of performing genetic testing for children with developmental disabilities continues to be controversial, and some have questioned whether the clinical utility of these tests justifies their expense. Moreover, a number of ethical concerns have been raised concerning the interpretation of variants of unknown significance, the identification of incidental genomic findings unrelated to the child's developmental condition, and the potential for genetic diagnoses to result in discrimination or increased stigmatization. $^{20-24}$

As clinical, technological, and ethical perspectives on genetic testing continue to evolve, research is needed to better understand how these tests are used in practice. To date, few studies have examined the rate at which children with developmental disabilities receive genetic testing. In a 2012 study of 154 parents of children with ASDs, 32\% reported that their child had undergone genetic testing. ${ }^{25}$ The reported prevalence of genetic testing was higher, at $41 \%$, in a larger 2014 survey of 397 parents of children with ASDs. ${ }^{26} \mathrm{~A}$ drawback of these studies is that they were based on convenience samples, which may limit the generalizability of their findings. Moreover, these studies restricted their analyses to children with ASDs and did not consider those with other developmental conditions. Thus, the aim of the present study was to assess the prevalence and correlates of genetic testing in a nationally representative sample of school-age US children with developmental conditions, including ASD, ID, and/or developmental delay (DD).

\section{Methods}

Data were analyzed from the 2011 Survey of Pathways to Diagnosis and Services ("Pathways"), a cross-sectional, telephone-based survey of the parents and guardians of US children with special health care needs (CSHCN) between the ages of 6 years and 17 years who had ever been diagnosed with developmental conditions, according to parent's report. ${ }^{27}$ The Pathways survey, which was conducted by the CDC's National Center for Health Statistics (NCHS) State and Local Area Integrated Telephone Survey (SLAITS) program, was a follow-up to the 2009-2010 National Survey of Children with Special Health Care Needs (NS-CSHCN). ${ }^{27}$ Respondents who had indicated in the NS-CSHCN that their child had ever been diagnosed with ASD, ID, and/or DD by a physician or other 
clinician were eligible for inclusion in the Pathways follow-up survey. Recontact was attempted for 6,090 randomly selected respondents out of the 7,572 eligible cases. ${ }^{27}$ Respondents who were successfully contacted were then asked to confirm that the child continued to meet the Pathways eligibility criteria. ${ }^{27}$ In total, 4,032 Pathways telephone interviews were completed. ${ }^{27}$ When appropriate survey weights are applied, estimates derived from the Pathways dataset are representative of the noninstitutionalized US population of CSHCN that ever had ASD, ID, or DD, according to parent's report. The NS-CSHCN and Pathways datasets are publicly available and can be linked to each other.

Respondents were included in the present study if they indicated that their child had ASD, ID, and/or DD at the time of the Pathways interview. The presence of these conditions was assessed based on parental response to questions about whether, to the best of their knowledge, the child currently had "autism or an ASD"; "an ID or mental retardation"; and/ or "a DD that affects his or her ability to learn." Respondents who reported that their child had been previously diagnosed with ASD, ID, or DD but no longer had any of these conditions at the time of survey administration were excluded. Respondents who had indicated in the NS-CSHCN that their child had been diagnosed with Down syndrome were also excluded, since these children were likely to have undergone genetic testing prenatally or at birth, rather than in response to developmental concerns. The analysis included 3,371 children who met these criteria.

Receipt of genetic testing was assessed based on a question about whether the child had ever gotten "a genetic screening to confirm a diagnosis or so that you could learn more about his/her conditions." Possible responses were "no," "yes," or "don't know." The weighted prevalence of testing was calculated for the entire sample, for all children with ASD, and for all children with ID. Respondents were then classified into five mutually exclusive condition groups: 1) those with current ASD, but not ID or DD (ASD only, $\mathrm{n}=492)$; 2) those with current ASD and ID, with or without DD (ASD + ID, n=292); 3) those with current ASD and DD, without ID (ASD + DD, n=616); 4) those with current ID, with or without DD and without ASD (ID without ASD, $n=528$ ); and 5) those with current DD, without ID or ASD (DD only, $n=1,419)$. In all, 24 children whose parents indicated that they did not know whether the child currently had one of the conditions were not assigned to any of the condition groups but were included in the aggregate analyses. The prevalence of genetic testing was computed separately for each of these groups.
Associations between genetic testing and demographic factors were examined within each of the five condition groups. Information about each child's race, ethnicity, and sex was originally collected in the NS-CSHCN. Data about household characteristics, including income as a percentage of the federal poverty level and the highest education level of any parent living with the child, were also derived from the NS-CSHCN. The child's age was recorded during the Pathways interview. Multivariable logistic regression models were used to assess the relationship between genetic testing and each of these variables (age, sex, household income, household education, and race/ethnicity), after controlling for other sociodemographic characteristics.

Logistic regression analyses were also used to compare the odds of receiving genetic testing between condition groups. Pairwise comparisons were made between all three ASD groups (ASD + ID vs ASD only, ASD + DD vs ASD only, and ASD + ID vs ASD + DD), between the ASD + ID and ID without ASD groups, and between the ASD + DD and DD only groups. To account for the potentially confounding effects of demographic differences, multivariable logistic regression models adjusted for age, sex, race/ethnicity, household income, and household education level.

The association between past genetic testing and use of other health-care services was also examined. Logistic regression analyses were used to determine whether children who had ever been treated by a developmental pediatrician (DP) were more likely to have undergone genetic testing compared to those who had never seen a DP. Similarly, the odds of genetic testing use were compared between those who had and had not been seen by a neurologist. History of medical specialist care was assessed based on two questions about whether the child had "ever worked with a DP to meet his/her developmental needs" or had "ever worked with a neurologist to meet his/her developmental needs." Logistic regression analyses were also used to determine whether the odds of having undergone genetic testing were higher for children who had received "all the treatments and services necessary to meet his/her developmental needs" and had seen "all the service providers needed to care for his/her developmental needs" within the previous 12 months. For all analyses, multivariable logistic regression models controlled for age, sex, race/ethnicity, household income, and household education level.

Survey weights were applied to account for the complex structure of the Pathways survey. Significance was set at the $P<0.05$ level. All analyses were carried out using SAS SURVEY procedures available in SAS Version 9.04 software. 


\section{Results}

The demographic characteristics of this sample are summarized in Table 1. According to parent's report, less than onethird $(31.7 \%)$ of all children in this sample - including $33.6 \%$ of all children with ASD and $43.3 \%$ of all children with ID - had ever undergone genetic testing (Table 2). In all, 3.2\% indicated that they did not know if genetic testing had ever been performed. Logistic regression analyses showed that children in certain condition groups were significantly more likely to have received genetic testing than others. Among those that had ASD, rates of genetic testing were highest for those with comorbid ID (52.1\%) and lowest for those who had neither ID nor DD (17.2\%; Table 2). After controlling for numerous demographic factors (age, sex, household income,

Table I Demographic characteristics

\begin{tabular}{ll}
\hline Demographic characteristic & Percent \pm SE \\
\hline Age, years & $50.8 \pm 1.7$ \\
$6-1 \mathrm{I}$ & $49.2 \pm 1.7$ \\
$12-17$ & \\
Income & $48.2 \pm 1.7$ \\
$\leq 200 \% \mathrm{FPL}$ & $29.3 \pm 1.6$ \\
$200 \%-400 \%$ FPL & $22.5 \pm 1.3$ \\
$>400 \%$ FPL & \\
Household education & $32.4 \pm 1.8$ \\
HS or less & $67.6 \pm 1.8$ \\
More than HS & \\
Sex & $70.4 \pm 1.5$ \\
Male & $29.6 \pm 1.5$ \\
Female & \\
Race and ethnicity & $61.5 \pm 1.8$ \\
White non-Hispanic & $15.9 \pm 1.3$ \\
Black non-Hispanic & $13.4 \pm 1.4$ \\
Hispanic &
\end{tabular}

Notes: The three race and ethnicity categories did not include participants who were indicated to be both white and black or belonging to a different racial group than white or black.

Abbreviations: SE, standard error; FPL, federal poverty level; HS, high school.

Table 2 Rates of genetic testing by condition group

\begin{tabular}{ll}
\hline Condition group & $\begin{array}{l}\text { Prevalence of genetic } \\
\text { testing }(\% \pm \text { SE) }\end{array}$ \\
\hline All respondents (ASD + ID + DD) & $31.7 \pm 1.6$ \\
All ASD & $33.6 \pm 2.2$ \\
All ID & $43.3 \pm 3.5$ \\
Mutually exclusive groups & \\
ASD + ID (with or without DD) & $52.1 \pm 5.3$ \\
ASD + DD (without ID) & $36.5 \pm 3.4$ \\
ASD only (without ID or DD) & $17.2 \pm 2.9$ \\
ID without ASD (with or without DD) & $38.4 \pm 4.4$ \\
DD only (without ASD or ID) & $28.2 \pm 2.4$ \\
\hline
\end{tabular}

Abbreviations: SE, standard error; ASD, autism spectrum disorder; ID, intellectual disability; DD, developmental delay.
Table 3 Group comparisons of genetic testing utilization rates

\begin{tabular}{lll}
\hline Condition groups & OR (95\% CI) & aOR $^{\mathbf{a}} \mathbf{( 9 5 \% ~ C I )}$ \\
\hline ASD groups & & \\
ASD + ID vs ASD only (ref) & $5.4(3.0-9.7)^{*}$ & $7.2(3.8-13.4)^{*}$ \\
ASD + DD vs ASD only (ref) & $2.7(1.7-4.5)^{*}$ & $2.8(1.7-4.7)^{*}$ \\
ASD + ID vs ASD + DD (ref) & $2.0(1.2-3.3)^{*}$ & $2.4(1.4-4.0)^{*}$ \\
ID groups & & \\
ASD + ID vs ID without ASD (ref) & $1.7(I .0-3.1)$ & $2.1(1.2-3.7)^{*}$ \\
DD groups & & \\
ASD + DD vs DD only (ref) & $1.5(1.0-2.1)^{*}$ & $1.8(1.2-2.8)^{*}$ \\
\hline
\end{tabular}

Notes: aAjusted for age, sex, race, ethnicity, household income, and household education level. *Significance.

Abbreviations: OR, odds ratio; aOR, adjusted odds ratio; ASD, autism spectrum disorder; ID, intellectual disability; DD, developmental delay; ref, reference group.

household education, race, and ethnicity), the odds of having ever undergone genetic testing were significantly higher for the ASD + ID (adjusted odds ratio [aOR] =7.2) and the ASD $+\mathrm{DD}(\mathrm{aOR}=2.8)$ groups, compared to those with ASD only (Table 3). Those with ASD and ID were also significantly more likely ( $\mathrm{aOR}=2.1$; Table 3 ) to have undergone genetic testing than those who had ID without ASD (52.1\% vs 38.4\%; Table 2). Similarly, genetic testing rates were higher for the ASD + DD group (aOR $=1.8$; Table 3 ) than for the DD only group (36.5\% vs $28.2 \%$; Table 2 ).

Multivariable logistic regression analyses were used to assess the demographic correlates of genetic testing in this sample (Table 4). For the ID without ASD, DD only, and ASD + DD groups, no associations were identified between prior genetic testing and age, sex, household income, household education level, or race/ethnicity. In the ASD + ID group, Hispanic children were significantly less likely $(\mathrm{aOR}=0.2)$ to have undergone genetic testing than those who were non-Hispanic white. Among children with ASD only, rates of genetic testing were higher $(\mathrm{aOR}=3.9)$ for those who came from households in which the highest level of education attained by any adult was high school or less compared to those from households with higher levels of parental educational attainment.

Past genetic testing was found to be associated with use of a number of other health care services (Table 5). Even after controlling for demographic factors, the odds of having undergone genetic testing were significantly higher for children who had ever been evaluated by a DP $(\mathrm{aOR}=3.1)$ or a neurologist ( $\mathrm{aOR}=3.7$ ), compared to those who had not been seen by these specialists. Rates of genetic testing were also higher for children who were reported to have seen all needed service providers $(\mathrm{aOR}=1.7)$ within the previous year. However, receipt of all needed treatments and services within the previous year was not found to be significantly associated with genetic testing. 
Table 4 Demographic correlates of genetic testing by condition group

\begin{tabular}{|c|c|c|c|c|c|}
\hline $\begin{array}{l}\text { Demographic } \\
\text { characteristic }\end{array}$ & $\begin{array}{l}\text { ASD only, aOR } \\
(95 \% \text { CI) }\end{array}$ & $\begin{array}{l}\text { ASD + ID, aOR } \\
(95 \% \mathrm{CI})\end{array}$ & $\begin{array}{l}\text { ASD + DD, aOR } \\
(95 \% \mathrm{CI})\end{array}$ & $\begin{array}{l}\text { ID without ASD, aOR } \\
(95 \% \mathrm{Cl})\end{array}$ & $\begin{array}{l}\text { DD only, aOR } \\
(95 \% \mathrm{Cl})\end{array}$ \\
\hline \multicolumn{6}{|l|}{ Age, years } \\
\hline $6-11$ & I.I (0.5-2.5) & $0.8(0.3-2.1)$ & I.2 (0.6-2.3) & $0.7(0.4-1.3)$ & I.2 (0.8-2.0) \\
\hline $12-17$ & Ref & Ref & Ref & Ref & Ref \\
\hline \multicolumn{6}{|l|}{ Income } \\
\hline$\leq 200 \% \mathrm{FPL}$ & $1.4(0.4-4.2)$ & $0.5(0.2-1.3)$ & $1.0(0.5-1.9)$ & $1.2(0.6-2.8)$ & $1.5(0.9-2.4)$ \\
\hline$>200 \% \mathrm{FPL}$ & Ref & Ref & Ref & Ref & Ref \\
\hline \multicolumn{6}{|l|}{ Education } \\
\hline HS or less & $3.9(1.1-13.8)^{*}$ & $1.9(0.6-6.2)$ & I.2 (0.5-2.9) & $0.6(0.3-1.3)$ & $1.6(0.9-2.7)$ \\
\hline More than HS & Ref & Ref & Ref & Ref & Ref \\
\hline \multicolumn{6}{|l|}{ Sex } \\
\hline Male & $0.6(0.2-2.0)$ & $0.3(0.1-1.0)$ & $1.0(0.4-2.5)$ & $0.7(0.4-1.4)$ & I.3 (0.8-2.2) \\
\hline Female & Ref & Ref & Ref & Ref & Ref \\
\hline \multicolumn{6}{|l|}{ Race/ethnicity } \\
\hline Hispanic & $0.2(0.0-1.4)$ & $0.2(0.0-0.9)^{*}$ & $0.9(0.3-2.2)$ & $1.0(0.4-3.0)$ & $2.0(0.9-4.3)$ \\
\hline Black, non-Hispanic & $1.5(0.3-8.0)$ & I.I $(0.3-4.6)$ & $1.2(0.4-3.7)$ & $1.9(0.7-5.7)$ & $1.4(0.7-2.7)$ \\
\hline White, non-Hispanic & Ref & Ref & Ref & Ref & Ref \\
\hline
\end{tabular}

Notes: a For each comparison, multivariable logistic regression models adjusted for all other demographic characteristics (age, sex, race, ethnicity, household income, and household education level). *Significance.

Abbreviations: ASD, autism spectrum disorder; aOR, adjusted odds ratio; ID, intellectual disability; DD, developmental delay; Ref, reference group; FPL, federal poverty level; HS, high school.

\section{Discussion}

A number of professional organizations - including the AAP, American Academy of Neurology, and ACMG - have advocated for the use of genetic testing as a routine part of the diagnostic workup for children with developmental disabilities. However, few studies have examined the extent to which these tests are actually used in this population of patients. The aim of this study was to examine the prevalence and correlates of genetic testing among US children with ASD, ID, and/or DD, based on data from the CDC's Survey of Pathways to Diagnosis and Services. To our knowledge, this represents the first study to assess the rate of genetic testing in a nationally representative sample of school-age children with developmental conditions.

Of the $>3,000$ children in the Pathways sample who had ASD, ID, and/or DD at the time of survey administration, less than one-third were reported to have ever undergone genetic testing. Among all children with ASD, the rate of genetic testing was $33.6 \%$. This finding aligns with the results of a 2012 study that reported that genetic testing had been performed in $31.6 \%$ of the children represented in their sample. ${ }^{25}$ However, the estimate derived from the Pathways sample is slightly lower than that reported by Cuccaro et al, who found that the rate of genetic testing in their sample was $41.2 \% .{ }^{26}$ This discrepancy may reflect the fact that the prior study was based on a convenience sample that was potentially subject to ascertainment bias, whereas the Pathways sample was designed to be nationally representative. Overall, the present study is consistent with previous ones in its finding that genetic testing is underutilized in this population of children.

Although the Pathways survey did not directly assess the reasons why children did or did not undergo genetic testing, a number of factors may have contributed to the low rate of genetic testing reported in this sample. Previous studies have identified a number of potential barriers to the use of genetic services in this population, including limited parental awareness of these tests, transportation issues, and concerns about cost. ${ }^{26,28}$ Notably, in the Pathways sample, children who were reported not to have seen all needed service providers within the previous year were significantly less likely to have undergone genetic testing than those who had seen all needed providers. This finding suggests that broader issues with accessing health care services may be associated with difficulties obtaining genetic testing. Apart from access issues, other factors, such as limited parental interest in these tests, may have contributed to the low prevalence of genetic testing. A recent qualitative study that conducted structured interviews with 42 parents of children with ASDs found that approximately one-fourth were opposed to ASD genetic testing, most commonly due to a lack of perceived benefit. ${ }^{28}$

In addition to the parental considerations discussed previously, the low rate of genetic testing reported in this sample may reflect physician noncompliance with published guidelines. ${ }^{29}$ Although the Pathways survey did not ask respondents 
Table 5 Associations between health-care use and genetic testing

\begin{tabular}{llll}
\hline Health-care use & $\begin{array}{l}\text { Answered } \\
\text { "yes": percent } \\
\text { that received } \\
\text { genetic testing }\end{array}$ & $\begin{array}{l}\text { Answered } \\
\text { "no": percent } \\
\text { that received } \\
\text { genetic testing }\end{array}$ & $\begin{array}{l}\text { aOR } \\
(95 \% ~ C l)\end{array}$ \\
\hline $\begin{array}{l}\text { Ever worked with } \\
\text { a neurologist? }\end{array}$ & $47.6 \pm 2.4$ & $23.1 \pm 2.0$ & $3.7^{*}(2.8-5.1)$ \\
$\begin{array}{l}\text { Ever worked with } \\
\text { a developmental }\end{array}$ & $48.7 \pm 3.0$ & $24.0 \pm 1.8$ & $3 . I^{*}(2.3-4.2)$ \\
$\begin{array}{l}\text { pediatrician? } \\
\text { Received all needed }\end{array}$ & $34.0 \pm 1.8$ & $29.6 \pm 3.4$ & $1.3(0.9-1.8)$ \\
$\begin{array}{l}\text { treatments/services? } \\
\text { (previous I2 mos) }\end{array}$ & & & \\
$\begin{array}{l}\text { Saw all needed } \\
\text { service providers? } \\
\text { (previous I2 mos) }\end{array}$ & $34.9 \pm 1.8$ & $25.4 \pm 3.6$ & $1.7^{*}(1.1-2.5)$ \\
\hline
\end{tabular}

Notes: ancludes all children that had ASD + ID + DD but not Down syndrome at the time of survey administration. 'Those that answered "no" represented the reference group for all aORs; adjusted for age, sex, race, ethnicity, household income, and household education level. *Significance; $P<0.05$.

Abbreviations: aOR, adjusted odds ratio; mos, months; ASD, autism spectrum disorder; ID, intellectual disability; DD, developmental delay.

to indicate what type of health care provider had ordered genetic testing, this study found that children who had ever been treated by DPs or neurologists were more than three times as likely as those who had not seen these specialists to have undergone genetic testing. However, among those who had been seen by DPs or neurologists, rates of genetic testing were still modest, with roughly half reporting that no testing had been performed. This raises the possibility that specialists may not be consistently ordering recommended genetic tests for children with developmental disabilities. Overall, the extent to which our findings reflect clinician nonadherence to published guidelines- versus the other factors discussed previously, such as a lack of parental interest in or access to these tests - is not clear.

Variations in physicians' routine clinical approach to evaluating different types of developmental disabilities may also have contributed to the large between-group discrepancies in the prevalence of genetic testing that were identified in this study. This issue was addressed in a recent study that asked 267 DPs and child neurologists to indicate what laboratory tests they would order in three hypothetical cases involving children with ASD and/or ID. ${ }^{30} \mathrm{~A}$ significantly higher proportion of respondents indicated that they would order genetic testing for a hypothetical child with ASD and ID than for one who had ASD without cognitive impairment (A Tchaconas, A Adesman, personal communication, 2016). These results mirrored our findings from the Pathways sample, in which rates of genetic testing were highest for those with ASD and comorbid ID (52.1\%) and lowest for the ASD only group
(17.2\%). After adjusting for demographic factors, the odds of having undergone genetic testing were more than seven times higher in the ASD + ID group than in the ASD only group. The low rate of genetic testing in the latter group may, to some extent, reflect evolving perspectives about the value of performing genetic testing in children who have ASD without comorbid cognitive impairment. Although a number of older guidelines recommended that genetic testing for children with ASDs be reserved primarily for those with comorbid GDD/ ID, more recent guidelines published by the AAP and ACMG have indicated that genetic testing should be offered to all individuals with an ASD., ${ }^{9,12,14,16}$ Nonetheless, due primarily to concerns about yield, there continues to be controversy as to whether routine genetic testing is warranted for individuals on the autism spectrum who are of normal intelligence. ${ }^{7,31}$

Finally, methodological issues cannot be excluded as a possible contributor to the low rates of genetic testing reported in this study. The prevalence of genetic testing was assessed based on parental responses to a single question in the Pathways survey, and no attempt was made to independently verify their claims. It is probable that some of the Pathways respondents incorrectly reported that their child had never undergone genetic testing. This could have occurred due to a lack of knowledge about the nature of the laboratory tests that were ordered for their child or due to the length of time that may have elapsed between the etiologic workup and the Pathways interview. Another possibility is that physicians may have performed genetic testing without informing parents, although this is not likely to have been a common occurrence. For these reasons, the prevalence rates reported in this study may underestimate the true rate of genetic testing in this population. Another limitation is that the Pathways survey did not assess what kinds of genetic testing were performed. Previous studies have shown that physicians frequently do not comply with published guidelines for performing genetic testing in children with developmental disabilities, often ordering outdated studies such as karyotyping while failing to obtain recommended tests such as CMA. ${ }^{30}$ Therefore, although approximately one-third of this sample was reported to have a history of any genetic testing, the number of children for whom all recommended genetic tests were ordered may be substantially lower.

Additional methodological considerations may also have affected our findings. First, information about each child's current developmental conditions (ASD, ID, and/or DD) was obtained based on parent's report and was not independently verified. In particular, the significance of the DD diagnosis in this context is somewhat unclear. Given that the term "DD" is typically used to refer to children younger than 5-6 years 
of age, it is surprising that such a large proportion of this sample of children between the ages of 6 years and 17 years was identified as having DD at the time of the Pathways interview. ${ }^{415}$ Moreover, although most of the guidelines cited previously refer to children with GDD - which is typically diagnosed based on the presence of significant delays ( $<2$ standard deviations below the mean) in two or more aspects of development - the Pathways survey defined DD as "any developmental delay that affects [the child's] ability to learn." This broad definition could have allowed for children with relatively mild or isolated learning impairments - some of whom might not have met the criteria for GDD - to be included in the Pathways sample. On the other hand, the DD only group may also have included children who had substantial cognitive impairment, despite parent's report that they did not have ID. These considerations highlight the limitations of relying on parent-reported diagnoses. In spite of these weaknesses, the use of a large, nationally representative sample of noninstitutionalized US children with ASD, ID, and/or DD represents a major strength of the present study.

This study found that the majority of school-age children with developmental disabilities in the Pathways sample had never received recommended genetic testing. Given that genetic diagnoses may facilitate more accurate family counseling and enable physicians to provide affected children with targeted medical surveillance and treatment, the low rate of genetic testing reported in this study is troubling. Further research is needed to examine the causes of these findings and to identify barriers to receipt of genetic testing among children with developmental disabilities. A better understanding of the factors that affect patients' access to these tests and physicians' decisions to order them may help to guide future efforts to ensure that children with developmental conditions receive all needed health-care services.

\section{Disclosure}

The authors report no conflicts of interest in this work.

\section{References}

1. Boyle CA, Boulet S, Schieve LA, et al. Trends in the prevalence of developmental disabilities in US children, 1997-2008. Pediatrics. 2011;127(6):1034-1042.

2. Zablotsky B, Black L, Maenner M, Schieve L, Blumberg S. Estimated prevalence of autism and other developmental disabilities following questionnaire changes in the 2014 National Health Interview Survey. Natl Health Stat Report. 2015;(87):1-21.

3. American Psychiatric Association. DSM-5 Intellectual Disability Fact Sheet. Washington, DC: American Psychiatric Association; 2013.

4. Tirosh E, Jaffe M. Global developmental delay and mental retardation - a pediatric perspective. Dev Disabil Res Rev. 2011;17(2):85-92.

5. Shaffer LG; American College of Medical Genetics Professional Practice and Guidelines Committee. American College of Medical Genetics guideline on the cytogenetic evaluation of the individual with developmental delay or mental retardation. Genet Med. 2005;7(9):650-654.

6. Feero WG, Guttmacher AE, Mefford HC, Batshaw ML, Hoffman EP. Genomics, intellectual disability, and autism. $N$ Engl $J$ Med. 2012;366(8):733-743.

7. El-Fishawy P, State MW. The genetics of autism: key issues, recent findings, and clinical implications. Psychiatr Clin North Am. 2010;33(1):83-105.

8. Betancur C. Etiological heterogeneity in autism spectrum disorders: more than 100 genetic and genomic disorders and still counting. Brain Res. 2011;1380:42-77.

9. Filipek P, Accardo P, Ashwal S, et al. Practice parameter: screening and diagnosis of autism, report of the Quality Standards Subcommittee of the American Academy of Neurology and the Child Neurology Society. Neurology. 2000;55(4):468-479.

10. Shevell M, Ashwal S, Donley D, et al. Practice parameter: evaluation of the child with global developmental delay, report of the Quality Standards Subcommittee of the American Academy of Neurology and the Practice Committee of the Child Neurology Society. Neurology. 2003;60(3):367-380.

11. Moeschler JB, Shevell M; American Academy of Pediatrics Committee on Genetics. Clinical genetic evaluation of the child with mental retardation or developmental delays. Pediatrics. 2006;117(6):2304-2316.

12. Johnson CP, Myers SM; American Academy of Pediatrics Council on Children With Disabilities. Identification and evaluation of children with autism spectrum disorders. Pediatrics. 2007;120(5):1183-1215.

13. Miller DT, Adam MP, Aradhya S, et al. Consensus statement: chromosomal microarray is a first-tier clinical diagnostic test for individuals with developmental disabilities or congenital anomalies. Am J Hum Genet. 2010;86(5):749-764.

14. Schaefer GB, Mendelsohn NJ; Professional Practice and Guidelines Committee. Clinical genetics evaluation in identifying the etiology of autism spectrum disorders: 2013 guideline revisions. Genet Med. 2013;15(5):399-407.

15. Moeschler JB, Shevell M, Saul RA; Committee on Genetics. Comprehensive evaluation of the child with intellectual disability or global developmental delays. Pediatrics. 2014;134(3):e903-e918.

16. American Academy of Pediatrics. Initial medical evaluation of a child diagnosed with an autism spectrum disorder. Caring for Children with Autism Spectrum Disorders: A Resource Toolkit for Clinicians. 2nd ed. 2013.

17. Lopez-Rangel E, Mickelson EC, Lewis MS. The value of a genetic diagnosis for individuals with intellectual disabilities: optimising healthcare and function across the lifespan. Br J Dev Disabil. 2008;54(2):69-82.

18. Coulter ME, Miller DT, Harris DJ, et al. Chromosomal microarray testing influences medical management. Genet Med.2011;13(9):770-776.

19. Riggs $\mathrm{E}$, Wain $\mathrm{K}$, Riethmaier $\mathrm{D}$, et al. Chromosomal microarray impacts clinical management. Clin Genet. 2014;85(2):147-153.

20. Miller DT. Genetic testing for autism: recent advances and clinical implications. Expert Rev Mol Diagn. 2010;10(7):837-840.

21. Driessnack M, Daack-Hirsch S, Downing N, et al. The disclosure of incidental genomic findings: an "ethically important moment" in pediatric research and practice. J Commun Genet. 2013;4(4):435-444.

22. Desale M, Worden LT, Cohen JS, Wilms Floet AM, Hoon AH. Diagnostic evaluation in children with developmental delay: a cautionary tale for genetic testing. Clin Pediatr. 2012;51(12):1208-1210.

23. Marchant GE, Robert JS. Genetic testing for autism predisposition: ethical, legal and social challenges. Houston J Health Policy. 2008;9:203-235.

24. Jordan BR, Tsai DFC. Whole-genome association studies for multigenic diseases: ethical dilemmas arising from commercialization - the case of genetic testing for autism. J Med Ethics. 2010;36(7):440-444.

25. Wydeven KV, Kwan A, Hardan AY, Bernstein JA. Underutilization of genetics services for autism: the importance of parental awareness and provider recommendation. J Genet Couns. 2012;21(6):803-813.

26. Cuccaro ML, Czape K, Alessandri M, et al. Genetic testing and corresponding services among individuals with autism spectrum disorder (ASD). Am J Med Genet A. 2014;164(10):2592-2600. 
27. Centers for Disease Control and Prevention NCHS SLAITS [webpage on the Internet]. 2011 Survey of Pathways to Diagnosis and Services Frequently Asked Questions; 2012. Available from: http:// www.cdc.gov/nchs/data/slaits/PathwaysFAQ.pdf. Accessed January 8, 2016.

28. Chen L-S, Xu L, Huang T-Y, Dhar SU. Autism genetic testing: a qualitative study of awareness, attitudes, and experiences among parents of children with autism spectrum disorders. Genet Med. 2013;15(4):274-281.
29. Peabody J, DeMaria L, Tamandong-LaChica D, Florentino J, Acelajado MC, Burgon T. Low rates of genetic testing in children with developmental delays, intellectual disability, and autism spectrum disorders. Global Pediatr Health. 2015;2:DOI: 2333794X15623717.

30. Tchaconas A, Adesman A. Medical evaluation of children with intellectual disability: clinician compliance with published guidelines. J Intellect Disabil Diagn Treat. 2015;3(2):97-109.

31. Chodirker BN, Chudley AE. Routine genetic testing for Asperger syndrome. Genet Med. 2008;10(11):843-845.

\section{Publish your work in this journal}

The Application of Clinical Genetics is an international, peer-reviewed open access journal that welcomes laboratory and clinical findings in the field of human genetics. Specific topics include: Population genetics; Functional genetics; Natural history of genetic disease; Management of genetic disease; Mechanisms of genetic disease; Counselling and ethical issues; Animal models; Pharmacogenetics; Prenatal diagnosis; Dysmorphology. The manuscript management system is completely online and includes a very quick and fair peer-review system, which is all easy to use. Visit http://www.dovepress.com/testimonials.php to read real quotes from published authors.

Submit your manuscript here: https://www.dovepress.com/the-application-of-clinical-genetics-journal 